MODELING, IDENTIFICATION AND CONTROL, 1993, VOL. 14, NO. 1, 43-58

doi:10.4173/mic.1993.1.3

\title{
On the use of constitutive internal variable equations for thermal stress predictions in aluminium casting
}

\author{
ASBJØRN MO† and ERIK J. HOLM $\ddagger$
}

Keywords: Internal variables, constitutive equations, thermal stresses, aluminium casting.

The report discusses the use of constitutive equations with internal variables in mathematical calculations of the thermally induced strains and stresses that arise during casting of aluminium. Application of the so-called MATMOD (MATerials MODel) constitutive relations for the viscoplastic deformation of the material is first discussed in relation to the specific characteristics of the casting process. Some numerical time integrations of the one-dimensional version of the MATMOD equations that are relevant for aluminium casting are then presented. The evolution of temperature and total strain are here chosen to be close to values experienced in the direct chill (D.C.) casting process, and values for thermally induced strains and stresses are calculated. The results are compared with predictions based on the traditional approach, in which a set of representative stress-strain curves at different constant temperatures and strain rates constitute the complete description of the viscoplastic deformation.

\section{Introduction}

Mathematical models that predict the evolution of temperature, stress, and strain fields in metals during industrial processing have become an important tool in obtaining a better understanding of the processes and in optimizing them. An important class of models is based on the mechanistic conservation laws for mass, energy, and momentum, and the quantitative description of the mechanical properties of the metal during processing is given through an appropriate set of constitutive equations.

In recent years the concept of internal variables has become an important part of modern constitutive theories. Internal variable descriptions have proved to be well suited to account for temperature and strain rate effects in a physically more realistic manner than the well-established plasticity theory used in most mathematical models of the above-mentioned class. The complete description of the viscoplastic deformation is, in the traditional theory, based on a set of representative stress-strain curves at different constant temperatures and strain rates, and no consideration is given to physical phenomena involved. Central in the new theories, on the other hand, is a constitutive equation that relates the total viscoplastic strain rate to the stress, the temperature, and some internal variables that represent the microstructural state of the material. Rate equations for the internal variables are also incorporated, and as time proceeds these are integrated to imitate the development of the microstructure during the processing of the material.

Received 26 March 1991.

† SINTEF SI, 0314 Oslo 3, Norway.

$\ddagger$ Institutt for Energiteknikk, 2007 Kjeller, Norway.

Reprinted with permission from Journal of Thermal Stresses, 1991, 14, 571-587. 
One of the basic ideas of the modern constitutive theories is that they can predict any kind of viscoplastic deformation without regard to any particular process for permanent deformation of the metal. A typical example is the set of so-called MATMOD (MATerials MODel) equations originally developed by Miller (1975). These equations probably attempt to cover the broadest set of phenomena, and different versions of them have been reviewed in Miller (1987). The breadth of phenomena that are to be quantified within the unification concept, however, comes in many cases at the expense of accuracy in the equations (Miller 1987, p. 139). On the basis of these considerations, a thorough study of the process characteristics and their impact on microstructural phenomena is necessary to select sufficiently accurate constitutive relations for a mathematical model of the process.

This investigation has been directed toward the casting of aluminium. An important problem in casting is the formation of thermally induced strains and stresses, which can lead to defects during or at the end of the process. This report therefore discusses the use of constitutive equations with internal variables in mathematical calculations of thermal stresses. We will furthermore compare results obtained by an internal variable description with thermal stress predictions based on the traditional concept of representative stress-strain curves.

Central to most mathematical models for predicting thermally induced strains and stresses is some functional dependence of the form $\bar{\sigma}=\bar{\sigma}\left(\bar{\epsilon}^{\mathrm{p}}, T\right)$, where $\bar{\sigma}$ is the flow stress, $\bar{\epsilon}^{\mathrm{p}}=\int d \bar{\epsilon}^{\mathrm{p}}$ is the accumulated effective plastic strain, and $T$ is the temperature. Examples can be found (Mathew and Brody 1976, Moriceau 1975) in which D.C. casting of aluminium is studied, and a review of this type of computations was presented by Dantzig (1989). These models also incorporate creep by using the so-called overlay concept (Owen and Hinton 1980), in which the total viscoplastic strain is subdivided into a time-independent plastic part and time-dependent creep. An objection to this concept is, however, that strain generated by creep is mechanically indistinguishable from that resulting from time-in-dependent plastic flow. In particular, at higher temperatures only the combined effect is experimentally observable (Thomas, Samarasekera and Brimacombe 1987, Zienkiewitcz and Cormeau 1974). Another method of incorporating the rate dependence of the flow stress is by replacing the relation $\bar{\sigma}=\bar{\sigma}\left(\bar{\epsilon}^{p}, T\right)$ by $\bar{\sigma}=\bar{\sigma}\left(\bar{\epsilon}^{p}, \bar{\epsilon}^{p}, T\right)($ Perzyna 1966$) . \bar{\epsilon}^{\mathrm{p}}$ is now the accumulated total viscoplastic strain, and $\overline{\bar{\epsilon}}^{\mathrm{p}}$ is the viscoplastic strain rate. A constitutive description similar to this was used in a model for thermal stress predictions in D.C. casting of aluminium billets developed by Fjær and Mo (1990).

In relationships of the form $\bar{\sigma}=\bar{\sigma}\left(\bar{\epsilon}^{\mathrm{p}}, T\right)$ or $\bar{\sigma}=\bar{\sigma}\left(\bar{\epsilon}^{\mathrm{p}}, \bar{\epsilon}^{\mathrm{p}}, T\right), \bar{\epsilon}^{\mathrm{p}}$ is tacitly regarded as a state variable that is representative of the current microstructure, and this gives rise to some fundamental problems. The relations are, in fact, only generalizations of the assumption of a universal stress-strain curve (see, for example, Malvern (1969)), which is a basic concept in the classical isothermal theory for isotropic work-hardening plastic materials. For casting, where isothermal conditions are manifestly not present, one cannot expect the effective plastic strain accumulated at some high temperature to be representative of the microstructure at a lower temperature. Constitutive relations based on this assumption should therefore be rejected.

Also, the isotropic hardening assumption is questionable in constitutive relations for thermal stress modeling because the material in some regions of the casting can be subjected to nonproportional straining. It can, for example, be clearly seen in aluminium D.C. casting computations that all viscoplastic strain rate components change sign locally in positions close to the water-impingement point, which is situated a few millimeters below the mould (Fjær and Mo 1990). 
The solidification of a cylindrical casting was studied by Smelser and Richmond (1988), who used the constitutive relations with one internal variable representing isotropic hardening as discussed in Sample and Lalli (1987). Internal variables are, however, not yet commonly used in mathematical models for thermal stress calculations in aluminium casting, and no reports have been found in which results obtained by internal variables are compared with predictions based on the traditional concept of representative stress-strain curves.

The development of constitutive equations to be used in mathematical process modeling consists of three parts. First, one has to find a pertinent set of equations suitable for the particular process. The second part is the experimental determination of the material constants, and the design of these experiments should be based on the constitutive description itself. A second kind of experiment is needed for the verification of the complete constitutive description used in connection with the specific process. This is the third part of the constitutive model development. In the present report we only deal with the first of these three parts. We do not, however, intend to give a detailed review of all the internal variable theories and their applicability for our thermal stress problem. Several of these theories have been discussed and compared (Miller 1987) from a more general point of view.

In the second section of this report, aluminium casting is considered in order to focus on the main characteristics that should be inherent in the constitutive equations. On the basis we then introduce the MATMOD equations in the third section and briefly discuss their application in thermal stress computations. In the last section we have solved one-dimensional MATMOD equations in computations quite relevant for the thermal stress problem. Comparisons with results obtained by a constitutive description of the viscoplastic deformation based on the well-established concept of representative stress-strain curves are also presented in this final section.

\section{Characteristics of aluminium casting}

The thermally induced elastic-viscoplastic deformation of metallic materials can normally be described within the geometrically linear theory (Dantzig 1989). This implies the necessity of a constitutive model with good accuracy for small strains. Also, the strain rates are small, having maximum values of approximately $10^{-2} \mathrm{sec}^{-1}$.

The constitutive relation between the flow stress and the (effective) viscoplastic strain rate depends strongly on temperature and on the ability of the dislocations to move. The development of the dislocation structure (i.e., the configuration and density of dislocations) of a specific alloy is in turn modeled through the strain hardening and recovery properties. The flow stress can furthermore depend on other microstructural characteristics such as grain size, density or solute atoms, and the density and coarseness of precipitates. We therefore proceed with a brief discussion of how the microstructural effects alter the mechanical properties of an aluminium alloy during casting and how these effects can be accounted for in the constitutive modeling of thermally induced viscoplastic strain.

\subsection{Temperature dependence}

Aluminium alloys experience a large range of temperatures in casting. The cooling rates vary from $0 \cdot 1^{\circ} \mathrm{C} \cdot \mathrm{sec}^{-1}$ in sand casting to $10^{\circ} \mathrm{C} \cdot \mathrm{sec}^{-1}$ in thin-walled die casting and D.C. casting, and in strip casting the cooling rate can even reach $1000^{\circ} \mathrm{C} \cdot \mathrm{sec}^{-1}$. As a result of relatively large temperature gradients, thermal contraction leads to significant elastic-viscoplastic deformation of the casting. Computations carried out 
by Fjær and Mo (1990) for a D.C. casting process show furthermore that considerable straining is present for all temperatures from the coherency temperature, $T_{\mathrm{c}}$, down to room temperature, $T_{\mathrm{r}}\left(T_{\mathrm{c}}\right.$ is defined as the temperature above which the metal is treated as a liquid and below which it is treated as a solid. For a more thorough discussion of this temperature, see (Claxton 1973).) One therefore needs a constitutive description for the viscoplastic straining of the material that is valid in the entire temperature range (i.e., $T_{\mathrm{r}} \leqslant T \leqslant T_{\mathrm{c}}$ ) and in which the microstructure development typical for the cooling of the alloy can be accounted for.

The mechanical properties of heat-treatable aluminium alloys depend strongly on the number and size of precipitates, and at temperatures below the solvus secondary precipitation becomes important if sufficient time for diffusion of solute elements is provided. A major problem in specifying the flow stress dependence on temperature is therefore to quantify the effect of such solid-state reactions. By comparing typical cooling rates for aluminium casting processes with a time-temperaturetransformation-diagram for precipitation of, for example, $\mathrm{Mg}_{2} \mathrm{Si}$ in 6000 -series alloys (Mondolfo (1976), p. 571), one finds that the cooling often is so rapid that the elements remain in solid solution during the entire cooling. Consequently, we shall in the present investigation simply neglect the effect of secondary precipitation because this obviously leads to less complexity of the constitutive model.

The above simplification restricts the description to non-heat-treatable alloys and to heat-treatable alloys subjected to a sufficiently rapid cooling. In the latter case this can lead to problems when numerical values for the coefficients in the constitutive equations are to be experimentally determined because this kind of experiment normally is carried out on time scales much longer than those characteristic for casting. To ensure that neither precipitation nor Gunier-Preston-zone formation becomes an important mechanism for the 'destruction' of the cast microstructure, the experiments must therefore only be carried out at temperatures above the solvus or at temperatures that are low enough to neglect the diffusion of elements.

As a result of these experimental considerations, we suggest the use of a constitutive model in which the temperature dependence of the equations can be interpolated from the upper temperature level to the lower. Mathematically this means that the temperature dependence should be an explicit variable of the constitutive equations instead of being introduced through temperature-dependent coefficients. Such equations have the important practical benefit of allowing predictions to be made of the behaviour at any desired temperature on the basis of data for other temperatures. Theories in which the temperature dependence is modeled in this manner have been discussed and compared by Bammann and Krieg (1987).

In the mushy zone $\left(T_{\mathrm{s}}<T<T_{\mathrm{b}}\right.$, where $T_{\mathrm{s}}$ is the solidus temperature and $T_{1}$ is the liquidus temperature), the interplay of the stress field in the solid with the interdendritic flow in the liquid is crucial (Smelser and Richmond 1988). Several authors have discussed the mechanistic conservation laws in the mushy zone, and in a recent report Ganesan and Poirier (1990) have derived macroscopic conservation equations for mass and momentum during solidification. In their approach, the mushy zone is regarded as two continuous, interpenetrating phases, and there is a general need for a constitutive model for the mushy zone that is based on a similar two-phase approach. In most mathematical models for thermal stress predictions only a one-phase model of the mushy zone is considered, and the stress distribution is assumed to be governed by Cauchy's equations for all temperatures below some coherency temperature $T_{\mathrm{c}}$. This obviously leads to somewhat inaccurate modeling for temperatures between $T_{\mathrm{c}}$ and $T_{\mathrm{s}}$, 
and the benefits of any two-phase constitutive model cannot be exploited. Development of an elaborate two-phase constitutive model for the mushy zone is beyond the scope of this report, but one can in the first instance simply extrapolate the explicit temperature functional dependence of a one-phase model up to $T_{\mathrm{c}}$.

\subsection{Strain hardening}

Strain hardening effects are due mainly to the development of a dislocation configuration. In constitutive models the phenomenon is normally divided into two different parts, namely isotropic and kinematic hardening, and these two parts are often modeled by two different internal variables representing the current hardness of the material. One variable is a positive scalar that accounts for the isotropic part, and the other is a tensor representing anisotropic effects developed by kinematic hardening. In many constitutive descriptions, only the isotropic part is taken into account. Such descriptions are therefore best suited for mathematical modeling of proportional straining of a material (i.e., processes for which the two hardening effects are experimentally indistinguishable). Kinematic hardening has, however, a considerable effect in processes at small strains because the number of mobile pile-up dislocations then is relatively large compared to the total number of dislocations. In casting processes where the strains are small and where the material, at least in some regions of the casting, is subjected to nonproportional straining, it is therefore necessary to discriminate between the two hardening effects.

\subsection{Recovery}

Whereas strain hardening represents an increase in the dislocation density and movement of mobile dislocations, recovery phenomena are characterized by annihilation of dislocations. This leads to negative terms in the rate equations for the internal variables representing the hardness of the material. Because recovery is thermally activated, these terms are functions of temperature and the current state of hardness. In problems with large plastic deformations the dislocation density will become high, and further straining of the material may enhance the annihilation of adjacent dislocations. In internal variable models this phenomenon is called dynamic recovery and is modeled through terms in the rate equations that come in addition to the static recovery terms referred to above. Because casting is characterized by small strains and thereby only a modest dislocation density, one can neglect dynamic recovery terms in the constitutive equations.

Recovery is mainly governed by the self-diffusion of aluminium atoms and the climb of dislocations. At lower temperatures static recovery is therefore slow, and one might expect that dynamic and static recovery would be of the same order of magnitude. Compared to the strain hardening effects at these low temperatures, the recovery effects are, however, so small that a somewhat inaccurate modeling of the phenomenon has no influence on the predictions of thermal stresses during casting.

\subsection{Other microstructural considerations}

The class of microstructural parameters that are constant during the deformation can be accounted for in the material description through alloy-dependent constants in the constitutive equations. From our discussion it should be clear that the flow stress dependence on the density of solute atoms and the density and coarseness of precipitates belong, or at least are assumed to belong, within this class. Also, the grain 
size is constant during casting, and neither recrystallization nor the formation of subgrains occurs in castings as a result of the low strains involved.

Miller (1987) discusses the above mentioned strengthening mechanisms along with others and suggests how several of them can be modeled with separate intrinsic internal variables. (Intrinsic means that the internal variable can be calculated directly from the current values of the temperature and the viscoplastic strain rate without any numerical integration of a rate equation.) Compared to the uncertainty introduced in the description by disregarding effects as the change of flow stress with density of solute atoms and with the precipitation configuration, this kind of refinement will not give a more accurate description in our thermal stress context. An introduction of more internal variables does also lead to more material constants, which may complicate the experimental procedures for constant determination.

\section{The MATMOD equations}

One set of constitutive internal variable equations in which our 'casting-motivated' requirements are fulfilled are Miller's MATMOD equations, to which some references already have been made. Several versions of the MATMOD equations exist, and the original MATMOD equations are described in detail in Miller $(1975,1976)$. In his review on MATMOD (Miller 1987), Miller discusses two main directions into which the model has been developed further. MATMOD IV is an extension capable of representing strain softening (Lowe 1983, Lowe and Miller 1984). Four internal variables are employed to provide separate representations of short- and long-range internal stresses and separate representations of homogeneously distributed dislocations and heterogeneous dislocation substructures. This complicates the constitutive model with a large number of material-specific constants to be experimentally determined. Because strain softening does not play an important part in casting, the detailed material description inherent in these equations is not believed to be necessary.

The other extension in MATMOD is the introduction of intrinsic internal variables accounting for mechanisms of general lattice strengthening and solute drag (Schmidt and Miller 1982, Henshall and Miller 1989). Application of intrinsic internal variables for thermal stress computations in casting has already received comment above, and the original MATMOD equations are sufficient for our purpose. (A minor change has been made by replacing the melting temperature $T_{\mathrm{m}}$ in Miller's equations by the coherency temperature $T_{\mathrm{c}}$ in accordance with the considerations described earlier.) The selected version contains two internal variables representing kinematic and isotropic strain hardening and recovery, and these will here be called $R$ and $D$, respectively. Although our investigation can be based on one-dimensional equations, the full threedimensional version of MATMOD is derived in (Miller 1975, 1987). The onedimensional MATMOD equation that relates viscoplastic strain rate $\dot{\epsilon}^{\mathrm{p}}$ to stress $\sigma$, temperature $T$, and the internal variables is

$$
\dot{\epsilon}^{\mathrm{p}}=B \cdot \Theta(T) \cdot\left\{\sinh \left[\left(\frac{|\sigma-R|}{D}\right)^{3 / 2}\right]\right\}^{n} \operatorname{sgn}(\sigma-R)
$$

where the sgn function is equal to +1 or -1 according to the positive or negative value of its argument. Rate equations for the time derivatives $\dot{R}$ and $\dot{D}$ are given by

$$
\dot{R}=H_{1} \dot{\epsilon}^{\mathrm{p}}-H_{1} B \cdot \Theta(T) \cdot\left[\sinh \left(A_{1}|R|\right)\right]^{n} \operatorname{sgn}(R)
$$


and

$$
\dot{D}=H_{2}\left|\dot{\epsilon}^{\mathrm{p}}\right|\left(C_{2}+|R|-\frac{A_{2}}{A_{1}} D^{3}\right)-H_{2} C_{2} B \cdot \Theta(T) \cdot\left[\sinh \left(A_{2} D^{3}\right)\right]^{n}
$$

respectively, and the temperature factor $\Theta(T)$ is related to the temperature by the expression

$$
\Theta(T)=\left\{\begin{array}{lr}
\exp \left\{\left[-\frac{Q}{0 \cdot 6 \cdot R_{B} T_{\mathrm{c}}}\right] \cdot\left[\ln \left(\frac{0 \cdot 6 \cdot T_{\mathrm{c}}}{T}\right)+1\right]\right\} & \text { for } T \leqslant 0 \cdot 6 \cdot T_{\mathrm{c}} \\
\exp \left\{-\frac{Q}{R_{B} T}\right\} & \text { for } T \geqslant 0 \cdot 6 \cdot T_{\mathrm{c}}
\end{array}\right.
$$

$R_{B}$ is here the gas constant, and the material constants are $Q\left(\operatorname{or}\left(Q / R_{B}\right), n, B, H_{1}, H_{2}, C_{2}\right.$, $A_{1}$, and $A_{2}$.

\subsection{Initial values}

When used in process simulations, the constitutive differential equations that govern the internal variables require initial values. A thermal stress computation in casting needs initial values, which here will be called $R_{0}$ and $D_{0}$, respectively, at the coherency temperature $T_{\mathrm{c}}$ (see $\$ 2.1$ and Fjær and Mo 1990). Initial values applied in the thermal stress problem are obviously not the same as those that are to be applied, for example, in a simulation of a tensile test on a cast specimen at room temperature for material constant determination. In the latter simulation the initial values for $R$ and $D$ should reflect the current structure of the cast metal, and this is necessarily influenced by the process history at the actual position in the casting from where the test specimen has been taken.

Miller (1975) argues that $R_{0}$ in annealed metal is 0 . This is likely because most annealed metals have equal tensile and compressive yield strengths. A similar symmetry argument is adopted for the casting problem. $D$, on the other hand, does not start out at $\mathbf{0}$ inasmuch as the material has finite initial strength. In a material that undergoes considerable strain hardening during a process, Miller (1975) discusses how $D_{0}$ can be determined from measurements of the initial strength at the initial temperature of the process and regards $D_{0}$ as an additional material constant for the particular process in question.

\subsection{Material constants}

Experimental procedures for the determination of material constants in MATMOD that include steady-state creep, cyclic stress-strain deformation, and tensile testing are described in Miller $(1975,1976)$. Because MATMOD was originally developed for the analysis of high-temperature structures, however, such as gas turbine blades and nuclear reactor components, we believe that several modifications of the experiments should be made. For casting, models need to apply specifically for the small strain and small strain rate regime. Experimental information obtained, for example, from cyclic stress-strain deformation with several cycles and large values of the effective strain is then unlikely to be important. This kind of deformation history does not occur in casting, and an accurate prediction of cyclic stress-strain deformation is therefore not necessarily required.

Experiments for the determination of MATMOD material constants particularly relevant for simulations of casting of an aluminium alloy have not yet been carried out. 
The material constants employed below are therefore identical to those proposed in Miller (1975), which are valid for annealed, high-purity aluminium. Even though these constants cannot be fully quantitatively accurate for the casting of an industrial alloy, they are sufficient for demonstrating the qualitative capabilities of the MATMOD internal variable description when this is applied in thermal stress modeling in casting processes. Miller's MATMOD material constants for high-purity aluminium are shown in Table 1.

\section{Computations relevant for aluminium casting}

Some numerical calculations relevant for aluminium casting have been carried out to show the adequacy of the MATMOD constitutive model. In these examples we have integrated one-dimensional constitutive equations with realistic evolutions of the temperature and total strain as input. This corresponds to a homogeneous distribution of the temperature, stress, and strain fields in uniaxial straining. This simplified onedimensional modeling gives qualitatively correct results that are sufficient input for a meaningful discussion of the use of internal variables in casting simulations.

In mathematical modeling of thermal stresses, a finite element solution leads to a set of equations in which the displacement field normally is taken as the primary unknown (see, for example, (Dantzig 1989, Fjær and Mo 1990)). Thermal stress calculations are carried out by a simultaneous solution of these equations and the time integration of the constitutive equations. The finite element equations disappear when the temperature and strain fields are homogeneous, and only the constitutive (and the compatibility) equations are left. Because of the small deformation theory, the total strain can be subdivided into an elastic, a thermal, and a viscoplastic (or inelastic) part, which in one dimension leads to the expression (In formulations based on the overlay concept, the inelastic part is further subdivided. See, for example, Dantzig (1989)).

$$
\epsilon=\epsilon^{\mathrm{e}}+\epsilon^{\mathrm{T}}+\epsilon^{\mathrm{p}}
$$

where the thermal strain $\epsilon^{\mathrm{T}}$ corresponds to the (known) thermal contraction of the material. Letting $\beta(T)$ denote the (temperature-dependent) coefficient of thermal expansion, the thermal strain is given by

$$
\epsilon^{\mathrm{T}}=-\int_{T}^{T_{\epsilon}} \beta(T) d T
$$

\begin{tabular}{lll}
\hline Quantity & Numerical value & Units \\
\hline$Q / R_{B}$ & 17865 & $\mathrm{~K}$ \\
$n$ & 4.04 & \\
$B$ & $1.25 \times 10^{9}$ & $\mathrm{sec}^{-1}$ \\
$H_{1}$ & 600 & $\mathrm{MPa}$ \\
$H_{2}$ & 10 & \\
$C_{2}$ & 0.69 & $\mathrm{MPa}$ \\
$A_{1}$ & 0.99 & $\mathrm{MPa}^{-1}$ \\
$A_{2}$ & $1.33 \times 10^{-3}$ & $\mathrm{MPa}^{-3}$ \\
$D_{0}$ & 2.33 & $\mathrm{MPa}$ \\
\hline
\end{tabular}

Table 1. MATMOD constants for high-purity aluminium. 
where $T_{\mathrm{c}}$ is the coherency temperature defined above. The elastic strain $\epsilon^{\mathrm{e}}$ is related to the stress by Hooke's law:

$$
\epsilon^{\mathbf{e}}=\frac{\sigma}{E(T)}
$$

where $E(T)$ is the temperature-dependent Young's modulus.

Time integrations of the constitutive equations given above and in Eqns. (5) to (7) are carried out in our examples. The temperature dependency of $\beta$ and $E$ are found in (Mondolfo 1976, pp. 61, 81-82), and the coherency temperature $T_{c}$ has been given the value $913 \mathrm{~K}\left(640^{\circ} \mathrm{C}\right)$ as in (Fjær and Mo 1990). Input values for the temperature and one component of the total strain tensor as functions of time have furthermore been produced by the temperature and thermal stress models described in Brobak, Fjær, Jensen and Mo (1991) and Fjær and Mo (1990). The D.C. casting process is here considered, and approximately $100 \mathrm{sec}$ of the intermediate part of the casting process has been simulated. Evolutions of temperature and total strain are taken from two material points in the casting, and these cases are referred to as A and B as indicated in Fig. 1. We have chosen the radial component of the strain tensor in both examples.

The employed integration method corresponds to that used in Fjær and Mo (1990). This implies an inversion with respect to the stress of the constitutive Eqn. (1), that is,

$$
\sigma=R+D\left[\sinh ^{-1}\left(\frac{\left|\dot{\epsilon}^{p}\right|}{B \cdot \Theta(T)}\right)^{1 / n}\right]^{2 / 3} \operatorname{sgn}\left(\dot{\epsilon}^{p}\right)
$$

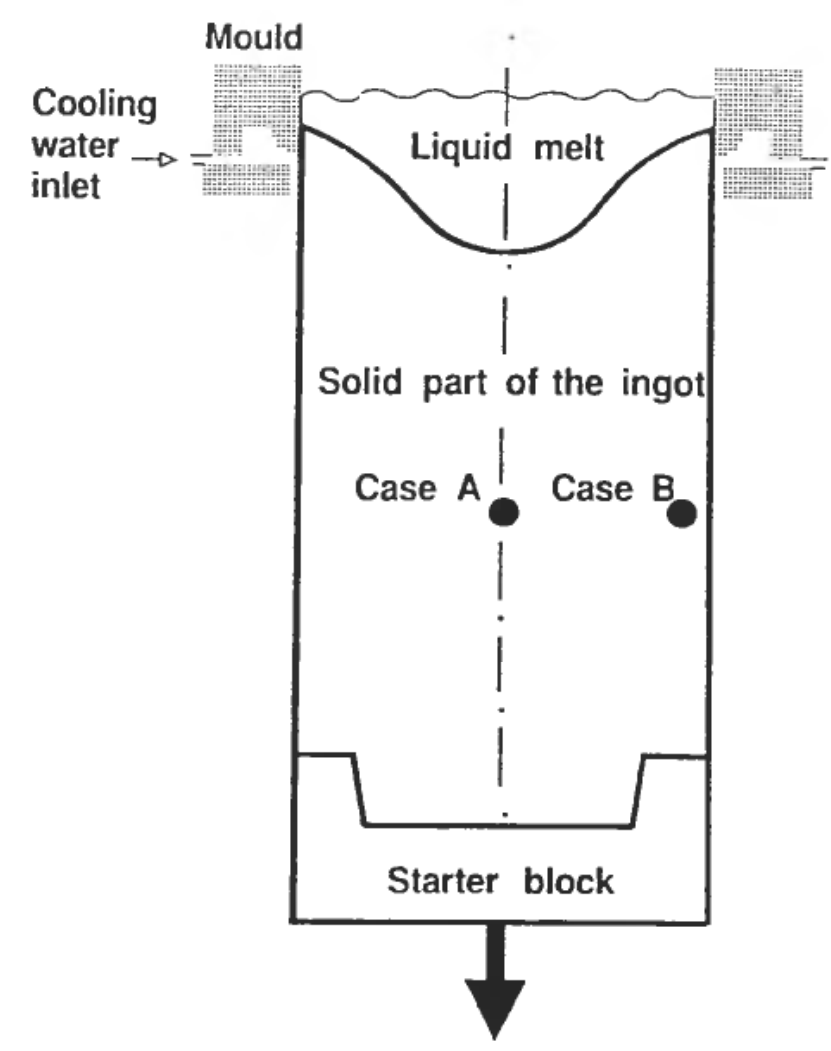

Figure 1. Material points in D.C. casting from which evolution of temperature and total radial strain are taken as input for cases $\mathbf{A}$ and $\mathbf{B}$. 
which is used instead of Eqn. (1) except when $\dot{\epsilon}^{\mathrm{p}}$ is close or equal to 0 . The inversion is due to the inherently stiff characteristics of the equations, particularly in the low strain rate sensitivity regime, where the derivative of $\dot{\epsilon}^{\mathrm{p}}$ with respect to $\sigma$ attains large values. (For more details, see (Bammann and Krieg 1987, Mo and Holm 1990).)

\section{MATMOD results}

Numerical predictions for cases A and B are shown in Figs. 2 and 3, respectively. One sees that the temperature evolution (which is input to the predictions) reflects the position in the billet: The temperature decreases more slowly in the centre of the casting (case A) than in case B, where the effect of the cooling water surrounding the ingot is stronger. In accordance with Eqn. (5) it should be noted that the total strain is subdivided into an elastic, a thermal, and a viscoplastic part and that the latter two parts have the same order of magnitude (maximum, $1 \%$ to $2 \%$ at room temperature).

It is evident from Figs. 2 and 3 that different temperature and total strain evolutions lead to quite different behaviour in viscoplastic strain and internal variables. For example, in case $\mathrm{A} \dot{\epsilon}^{\mathrm{p}}$ is almost negligible after approximately $60 \mathrm{sec}$ casting time or, accordingly, when the temperature is below approximately $230^{\circ} \mathrm{C}$. This leads to almost stationary values for $R$ and $D$ and thereby no strain hardening at lower temperatures. In case $\mathbf{B}$, on the other hand, $\dot{\epsilon}^{\text {p }}$, and thereby $\dot{R}$ and $\dot{D}$, have significant values in the entire temperature interval during the process.

There is nonproportional straining in case $B$, which can be seen from the shift in sign in $\dot{\epsilon}^{p}$. At higher temperatures it turns out that most of the strain hardening is due to kinematic hardening. It should furthermore be noticed that the change of sign in $R$ reflects a Bauschinger effect in the material. Kinematic hardening plays an important part also in case A, even though we here only have proportional straining. In both cases the kinematic hardening comes in addition to the isotropic hardening.

All variables $T, \dot{\epsilon}^{p}, R$, and $D$ determine the stresses. It is also important to have in mind that according to Eqn. (8) $\sigma$ is proportional to $D$, whereas it is related to $R$ in an additive manner. It is therefore convenient to use different scales on the axis for $R$ and $D$ in Figs. $2(d), 2(e), 3(d)$, and $3(e)$.

We finally notice that the sinh term and the term $H_{2}\left|\dot{\epsilon}^{\mathrm{p}}\right| A_{2} / A_{1} D^{3}$ in Eqn. (3) for $D$ both are negligible in the present calculations. This means that recovery in these particular examples is governed by the hyperbolic sine term in Eqn. (2) for $\dot{R}$, which is the kinematic contribution to the recovery effects.

\subsection{Comparisons with the classical theory}

Our MATMOD predictions have been compared with results obtained by the classical theory for elastic-viscoplastic materials based on representative stress-strain curves at different constant temperatures and strain rates. Central to the traditional description is a relation between the stress, the viscoplastic strain rate, the temperature, and the accumulated effective viscoplastic strain (Perzyna 1966, p. 296). This relation can in one dimension be written generally as

$$
\sigma=\sigma\left(\bar{\epsilon}^{\mathrm{p}},\left|\dot{\epsilon}^{\mathrm{p}}\right|, T\right) \operatorname{sgn}\left(\dot{\epsilon}^{\mathrm{p}}\right) \quad \text { where } \bar{\epsilon}^{\mathrm{p}}=\int\left|d \epsilon^{\mathrm{p}}\right|
$$

We notice that the accumulated effective viscoplastic strain is used as a state variable and that only isotropic strain hardening and recovery are incorporated in the theory.

MATMOD-produced stress-strain curves at different constant temperatures and strain rates are shown in Fig. 4, and results similar to these have been used as empirical input to the stress-strain curve-based constitutive approach. We have in Fig. 4 focused 


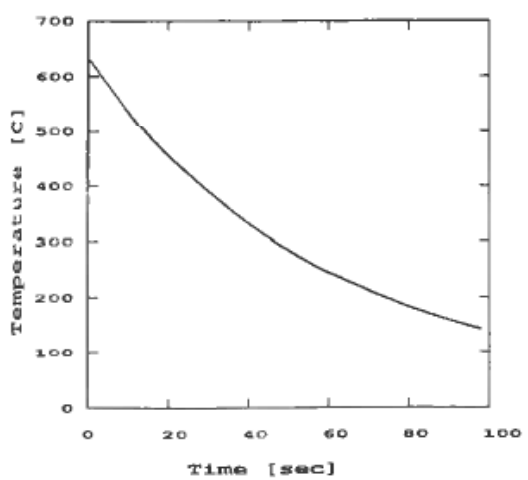

(a)

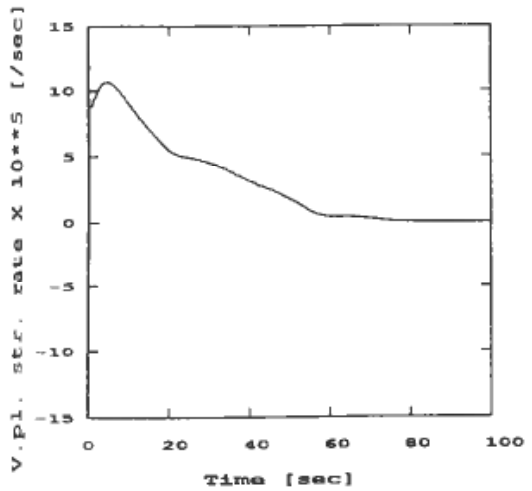

(c)

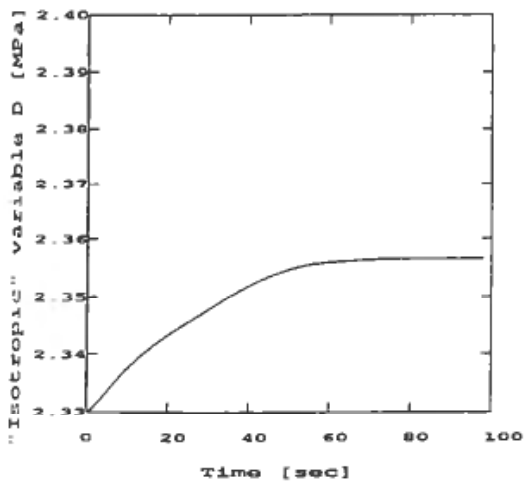

(e)

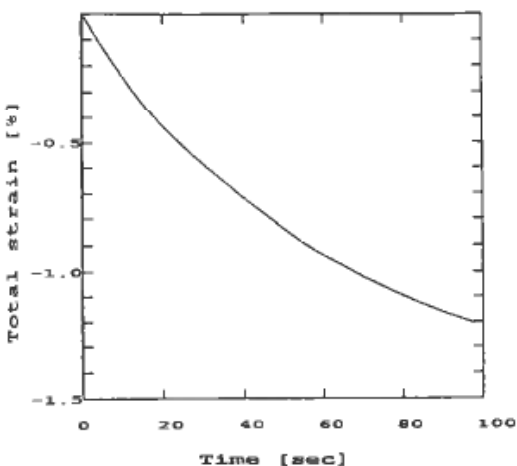

(b)

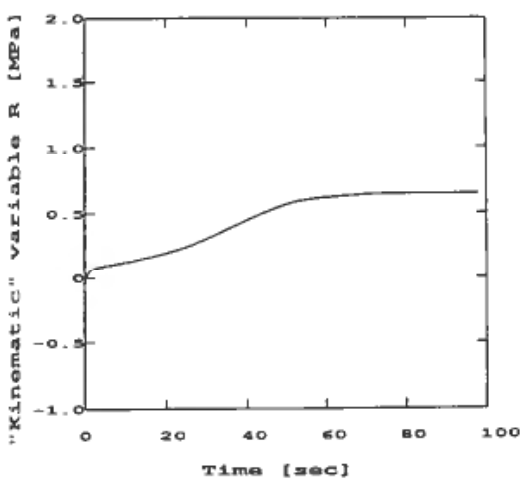

(d)

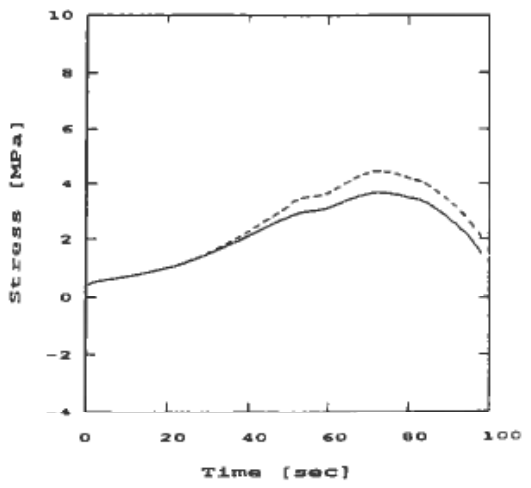

$(f)$

Figure 2. Evolution of temperature $T(a)$, total strain $\epsilon(b)$, viscoplastic strain rate $\dot{\epsilon}^{\mathrm{P}}(c)$, kinematic variable $R(d)$, isotropic variable $D(e)$, and stress $\sigma(f)$ in case A. The dashed line in $(f)$ corresponds to stresses obtained by the classical theory. 


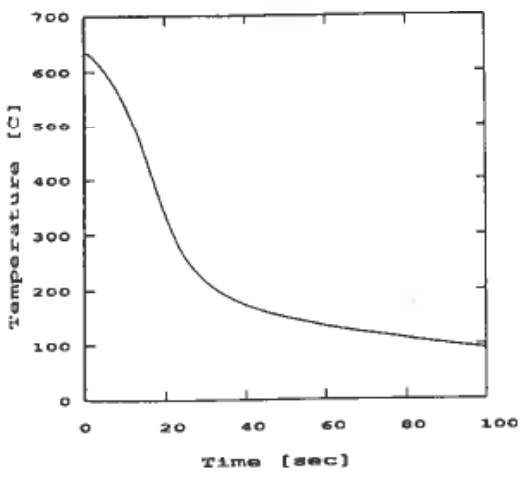

(a)

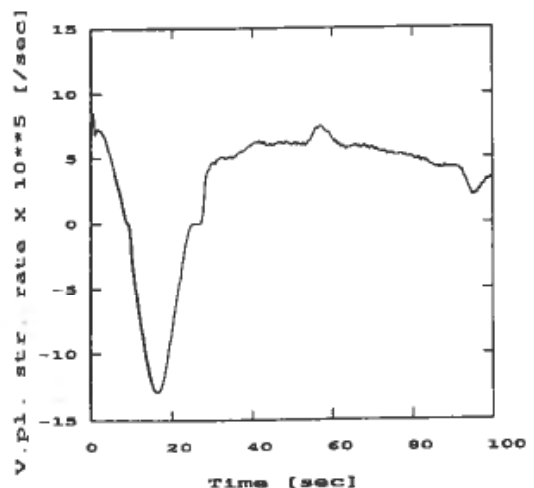

(c)

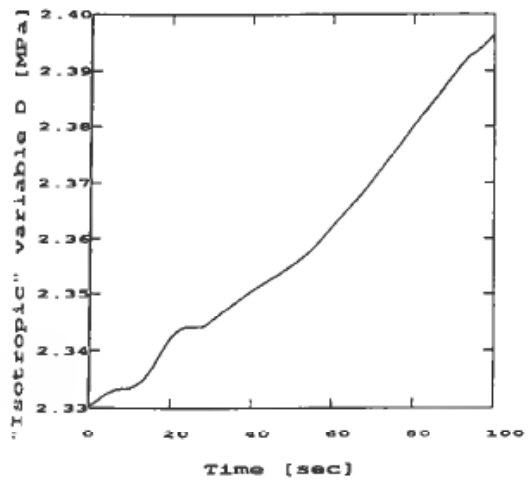

(e)

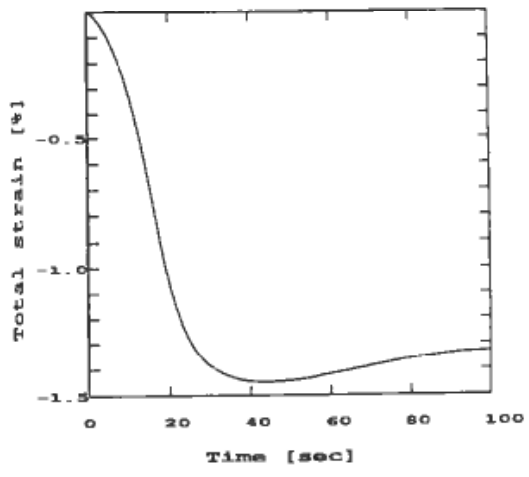

(b)

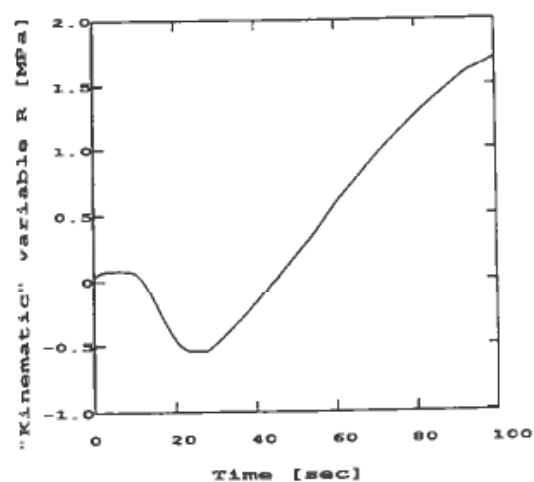

(d)

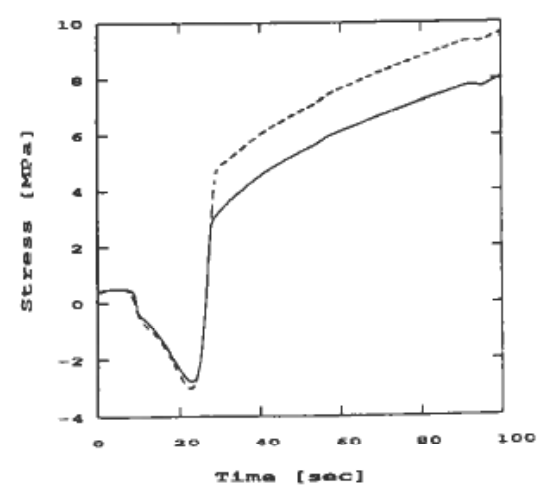

(f)

Figure 3. Similar as in case A for case B. 

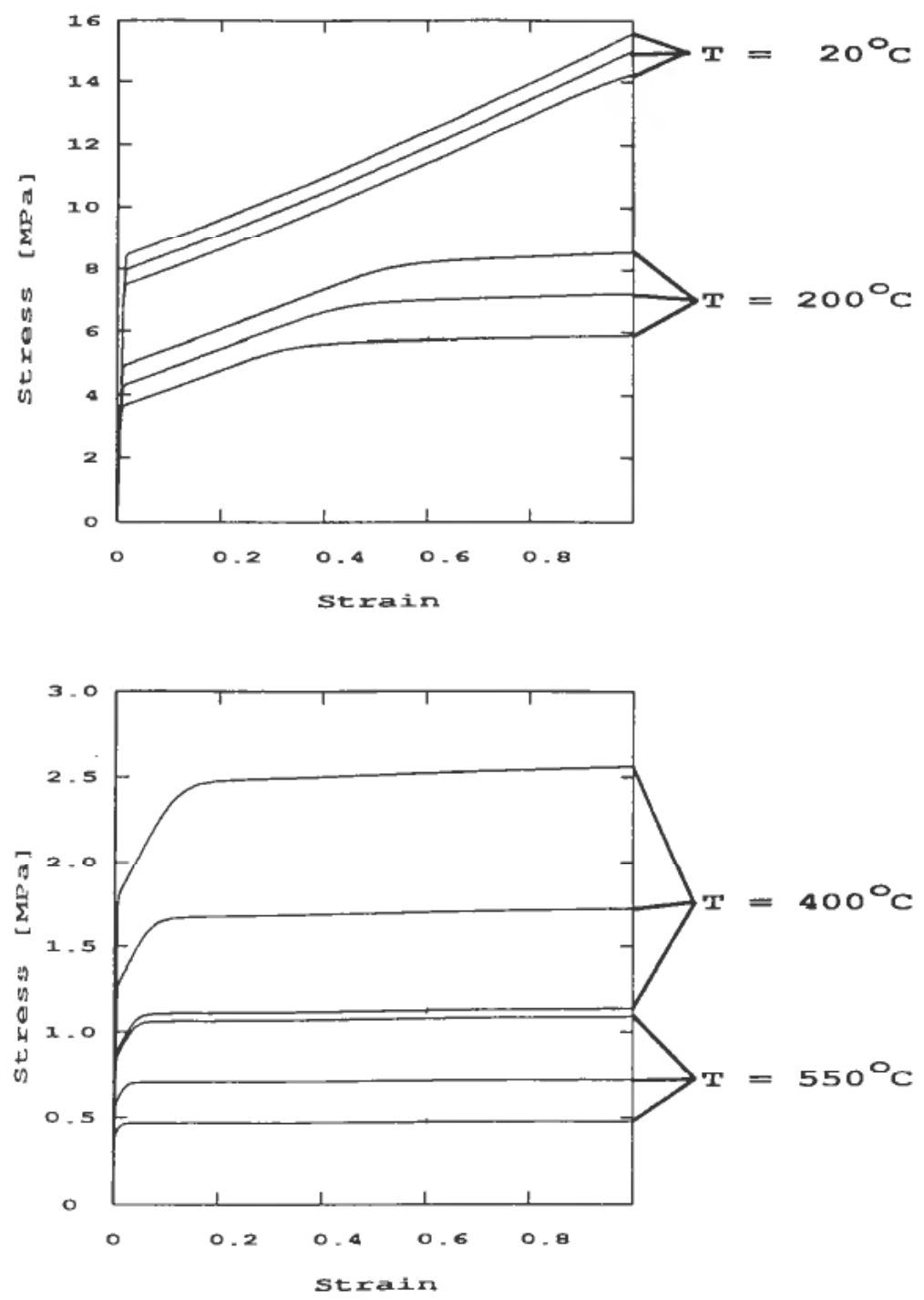

Figure 4. MATMOD-produced stress-strain curves for temperatures $20^{\circ} \mathrm{C}, 200^{\circ} \mathrm{C}, 400^{\circ} \mathrm{C}$, and $550^{\circ} \mathrm{C}$ and viscoplastic strain rates $10^{-5} \mathrm{sec}^{-1}, 10^{-4} \mathrm{sec}^{-1}$, and $10^{-3} \mathrm{sec}^{-1}$ (increasing stress values for each temperature correspond to increasing values of viscoplastic strain rate).

on the particular regimes of temperature, strain, and strain rate that are typical for casting, and it is seen that the curves do not have the typical shape expected in uniaxial straining up to $1 \%$ aluminium. We emphasize, however, that as long as the stress-strain curves are qualitatively correct, and as long as the same curves are input to the classical predictions as those that can be produced by MATMOD, our comparison will be valid.

In the thermal stress calculations, empirical stress-strain results are transferred to Eqn. (9) simply by integrating the MATMOD Eqns. (2), (3), and (8) with the actual values for $T$ and $\dot{\epsilon}^{\mathrm{p}}$ kept constant. $\sigma$ is then obtained when the current value of $\bar{\epsilon}^{\mathrm{p}}$ is reached. The stress-strain curves used in the thermal stress modeling based on the 
traditional method have, in other words, been produced by MATMOD. Any differences in the presented thermal stress results obtained by MATMOD and the classical approach are consequently due to the theoretical fundament of the two constitutive descriptions and not to any inaccuracy in a numerical interpolation between different stress-strain curves. It should also be noticed that in the comparison we implicitly assume that stress-strain results can be regarded as representative for all actual temperatures in the casting process. As stated earlier, one cannot generally expect this to be the case in the intermediate temperature regime when heat-treatable alloys are studied. Consequently, the need for an internal variable model is then even larger because an interpolation between high- and low-temperature experimental input fot the material behaviour may be required.

From Figs. $2(f)$ and $3(f)$ it is seen that the classical theory predicts larger absolute values for the stresses than the MATMOD theory, and we notice that the differences in stresses are largest at lower temperatures. This result reflects the flow stress dependence on $\bar{\epsilon}^{\mathrm{p}}$ in the description based on stress-strain curves. For example, in case A we see that $\dot{\epsilon}^{\mathrm{p}}$ has a negligible value below approximately $230^{\circ} \mathrm{C}$, which means that $\bar{\epsilon}^{\mathrm{p}}$ has obtained its value solely above this temperature. It should also be noticed that $\dot{\epsilon}^{\mathrm{P}}$ does not change sign in case A, which means that the results have not been disturbed by any kinematic recovery effects. In other words, as a result of the effect of thermal recovery at higher temperatures, we cannot regard $\bar{\epsilon}^{\mathrm{p}}$ as representative for the state of the material at lower temperatures.

The importance of incorporating kinematic hardening is revealed in Figs. $3(d)$ and $3(f)$. During the first $40 \mathrm{sec}$ of the process in case B, the internal variable $R$ passes through a history of being positive, then having a negative value, and finally becoming positive once more. This leads to smaller absolute values for the MATMOD-predicted stresses than in the traditional, isotropic theory, and the importance of modeling the Bauschinger effect in the material is clearly demonstrated.

Another kinematic effect can be seen by comparing the two cases A and B. In the former there is proportional straining during the entire process, but in case B there is nonproportional straining at higher temperatures. It turns out that the differences in stresses predicted by the different theories are larger in the nonproportional case than in the proportional case. This result is in accordance with the fact that kinematic and isotropic hardening are experimentally indistinguishable in processes with nonproportional straining (as discussed earlier). Because the material description in the classical theory is based solely on unidirectional tensile testing, one therefore expects that the nonproportional case B should reveal the largest differences in stresses.

\section{Conclusions}

The use of a constitutive internal variable model for mathematical modeling of thermally induced elastic-viscoplastic deformation in casting of aluminium alloys has been discussed. The benefits of modern internal variable equations are emphasized, and we have discussed how the MATMOD constitutive description can be applied in our particular context.

In comparisons between predictions obtained by the traditional stress-strain curve approach and the MATMOD theory, we have demonstrated serious shortcomings of the former constitutive description of rate-sensitive plastic materials. The most important of these shortcomings are that the accumulated effective (visco)plastic strain is regarded as a state variable and that kinematic hardening and recovery are neglected. 


\section{ACKNOWLEDGMENTS}

This research is funded by Hydro Aluminium, Elkem Aluminium, and the Royal Norwegian Council for Scientific and Industrial Research (NTNF). The authors have had numerous discussions pertaining the metallurgical basics of the paper with J. E. Tibballs, C. Simensen, and Y. Langsrud, all at SINTEF SI. Dr Tibballs has also corrected the manuscript, and we sincerely thank each of our colleagues for sharing their insights with us. We are furthermore grateful for stimulating discussions with H. G. Fjær at Institutt for energiteknikk.

\section{REFERENCES}

Bammann, D. and Krieg, R. D. (1987). Unified Constitutive Equations, Chapter 7 (Elsevier Applied Science, New York).

BrobaK, T. J., FJÆR, H. G., JenSEN, E. K. and Mo, A. (1991). Mathematical Predictions of Center Crack Formation in D.C. Casting of Aluminium Billets, Light Metals, TMS-AIME, Warrendale, PA, USA.

Claxton, R. J. (1973). Aluminium alloy coherence, Continuous Casting, 341-352 (AIME Metallurgical Society, New York).

DANTZIG, J. A. (1989). Thermal stress development in metal casting processes, Metall. Sci. Technol., 7, 133-178.

FJÆR, H. G., and Mo, A. (1990). ALSPEN-A mathematical model for thermal stresses in D.C. casting of aluminium billets, Metall. Trans., 21 B, 1049-1061.

Ganesan, S. and Poirer, D. R. (1990). Conservation of mass and momentum for the flow of interdendritic liquid during solidification, Metall. Trans., 21 B, 173-181.

Henshall, G. A. and Miller, K. (1989). The influence of solutes on flow stress plateaus, with emphasis on back stresses and the development of unified constitutive equations, Acta Metall., 37, 2693-2704.

Lowe, T. C. (1983). New Concepts in Modelling Strain Softening, Thesis, Stanford University, Stanford, California.

LOWE, T. C. and MiLLER, A. (1984). Improved constitutive equations for modelling strain softening - Part 1: Conceptual Development, J. Eng. Mat. Technol., XX, 337-348.

MALVERN, L. E. (1969). Introduction to the Mechanics of a Continuous Medium (Prentice-Hall, Englewood Cliffs, New Jersey).

MATHEW, J. and BRODY, H. D. (1976). Simulation of thermal stresses in continuous casting using a finite element method. Nucl. Metall., 20, 978-990.

Miller, A. K. (1975). A Unified Phenomenological Model for the Monotonic, Cyclic, and Creep deformation of strongly Work-Hardening Materials, Thesis, Stanford University, Stanford, California.

Miller, A. (1976). An inelastic constitutive model for monotonic, cyclic, and creep deformation: Part 1 - equations development and analytical procedures, J. Eng. Mat. Technol., XX, 97-105.

Miller, A. K. (1987). Unified Constitutive Equations, Chapter 3 (Elsevier Applied Science, New York).

Mo, A. and Holm, E. J. (1990). On the Use of Constitutive Internal Variable Equations for Mathematical Modelling of Thermal Stresses in D.C. Casting of Aluminium. Technical Report IFE/KR/F-90/095 and SI/890219-4, Institutt for Energiteknikk (IFE) and SINTEF SI (SI), Norway, July 1990.

Mondolfo, L. F. (1976). Aluminium Alloys, Structure and Properties (Butterworths, London).

MoriCEAU, J. (1975). Thermal stresses in D.C. casting of Al alloys, Light Metals, 2, 119-133, TMS-AIME, Warrendale, Pennsylvania.

Owen, D. R. J. and Hinton, E. (1980). Finite Elements in Plasticity (Pineridge Press Limited, Swansea, UK).

Perzyna, P. (1966). Fundamental problems in viscoplasticity, Adv. App. Mech., 9, 243-377.

Sample, V. M. and LaLli, L. A. (1987). Effects of thermomechanical history on hardness of aluminium, Mat. Sci. Technol., 3, 28-35.

SCHMIDT, C. G. and Miller, A. K. (1982). Overview 16: The effect of solutes on the strength and strain hardening behaviour of alloys, Acta Metall., 30, 615-625. 
SmFiser, R. E. and RICHMOND, O. (1988). Constitutive model effects on stresses and deformations in a solidifying circular cylinder, Modeling and Control of Casting and Welding Processes IV, 313-328, TMS, Warrendale, Pennsylvania.

Thomas, B. G., Samarasekera, I. V. aid Brimacombe, J. K. (1987). Mathematical model of the thermal processing of steel ingots: Part II. Stress model, Metall. Trans., 18 B, 131-147.

ZIENKIEWITCZ, O. C., and CORMEAU, I. C. (1974). Visco-plasticity-plasticity and creep in elastic solids-A unified numerical solution approach, Int. J. Numer. Meth. Eng., 8, 821-845. 